\title{
Can Computers Discover Ideal Knots?
}

\author{
Eric J. Rawdon
}

\section{CONTENTS}

1. Introduction

2. Background Information

3. Inscribing Smooth Knots in Polygons

4. Characterization Theorems for $R(K)$

5. Characterization Theorems for $R(P)$

6. The Main Theorem

7. Computation of Upper Bounds on Smooth Ropelength

8. Limiting Behavior

9. Discussion

Acknowledgments

References
2000 AMS Subject Classification: Primary 57M25

Keywords: Polygonal knots, geometric knots, ropelength
We discuss the relationship between polygonal knot energies and smooth knot energies, concentrating on ropelength. We show that a smooth knot can be inscribed in a polygonal knot in such a way that the ropelength values are close. For a given knot type, we show that polygonal ropelength minima exist and that the minimal polygonal ropelengths converge to the minimal ropelength of the smooth knot type. A subsequence of these polygons converges to a smooth ropelength minimum. Thus, ropelength minimizations performed on polygonal knots do, in fact, approximate ropelength minimizations for smooth knots.

\section{INTRODUCTION}

Many researchers have defined energy functions on smooth and polygonal knots. One of the initial objectives of mathematicians was to find a canonical flow from any unknot to a planar circle. In theoretical terms, this goal has not been realized; however, in practice, several polygonal energy functions have been successful in flowing very complicated unknots to a circle.

Knot energies have also become of increasing interest to scientists. Energy minimizing conformations capture information related to observed physical knotting and the statistical behavior of large ensembles of knots. For example, the average crossing number of ropelength minimized conformations is nearly linearly related to the gel speed of some DNA knots under certain experimental conditions [Katritch et al. 96, Stasiak et al 96, Stasiak et al. 98]. Furthermore, the Möbius energy and ropelength energy of minimized conformations (and their respective writhes) appear to be quantized over different families of knots [Cerf and Stasiak 00, Pierański and Przbyl 01, Hoidn et al. 02].

It is difficult to produce energy minimizing smooth curves. The only explicit description of nontrivial energy minimizing curves is in [Cantarella et al. 02], where the authors describe ropelength minimizing $C^{1,1}$ conformations for a class of simple links. Typically, one relies on computer simulation to flow knots to nearly optimal

(c) A K Peters, Ltd. 1058-6458/2003 \$0.50 per page Experimental Mathematics 12:3, page 287 
conformations. This is possible with the use of polygonal energies, which attempt to approximate the behavior of their smooth counterparts. The energy optimizing polygons are assumed to be similar to energy optimizing smooth knots. However, there are no theorems which prove that optimal polygons converge to optimal smooth curves. We prove this result for the ropelength energy.

We concentrate on the ropelength defined for $C^{2}$ knots in [Litherland et al. 99] (and subsequently extended to $C^{1,1}$ knots in [Durumeric et al. 97, Gonzalez and Maddocks 99, Cantarella et al. 02]) and the polygonal ropelength defined in [Rawdon 98, Rawdon 00]. To distinguish between the quantities, throughout this paper smooth always means $C^{1,1}$ and smooth ropelength is ropelength defined on $C^{1,1}$ knots. Similarly, polygonal ropelength means ropelength defined on piecewise-linear knots. Section 2 contains background information on the smooth and polygonal ropelength energies. In Section 3 , we describe the algorithm for inscribing $C^{1,1}$ knots in polygons that is used throughout the paper. Sections 4 and 5 contain new characterization theorems for the smooth and polygonal injectivity radius. These are used to prove the main theorem in Section 6: The injectivity radius of a polygon and its inscribed smooth knot are close. In Section 7, we compute upper bounds for the ropelength of smooth knot types. In Section 8, we show that the ropelengths of $n$-edge polygonal optima converge to the minimal ropelength of a smooth knot type as $n \rightarrow \infty$ and that a subsequence of the polygonal ropelength minima converges to a smooth ropelength minimum.

\section{BACKGROUND INFORMATION}

Given two feet of one-inch radius rope, is it possible to tie a nontrivial knot? Smooth ropelength was defined in [Buck and Orloff 95, Litherland et al. 99] to try to answer this question. Litherland et al. showed that one needs at least $5 \pi$ inches of idealized rope to tie a nontrivial knot. Research in [Cantarella et al. 02] and Diao [Diao 03] improved this bound to $2 \pi(2+\sqrt{2}) \approx 21.45$ and 24 , respectively. However, computer simulations suggest that one needs $\approx 32.76$ inches to tie a trefoil [Katritch et al. 96, Stasiak et al. 98, Millett and Rawdon 03].

Many ropelength energies have been defined for smooth curves [Krötenheerdt and Veit 76, Diao et al. 97, Kusner and Sullivan 98, O'Hara 98, Diao et al. 98a, Diao et al. 99, Gonzalez and Maddocks 99, Durumeric et al. 97] and for polygons [Katritch et al. 96, Stasiak et al 96, Kusner and Sullivan 98, Rawdon
98, Diao et al. 99, Gonzalez and Maddocks 99, Rawdon 00]. For this paper, we concentrate on the smooth ropelength energy defined in [Litherland et al. 99] and the polygonal ropelength defined in [Rawdon 98, Rawdon 00]. The smooth ropelength energy models rope as a non-selfintersecting tube with a knot as its core. For completeness, we include these definitions and some properties of these energies.

Definition 2.1. For a $C^{1} \operatorname{knot} K$ and $x \in K$, let $D_{r}(x)$ be the disk of radius $r$ centered at $x$ lying in the plane normal to the tangent vector at $x$. Let

$R(K)=\sup \left\{r>0: D_{r}(x) \cap D_{r}(y)=\emptyset\right.$ for all $\left.x \neq y \in K\right\}$.

The quantity $R(K)$ is called the injectivity radius or thickness radius of $K$. Define the ropelength of $K$ to be

$$
\rho(K)=\operatorname{Length}(K) / R(K),
$$

where Length $(K)$ is the arclength of $K$.

The injectivity radius is the radius of a thickest tube that can be placed about the knotted core without selfintersection. Note that in some of the literature, the thickness refers to the diameter of this thickest tube, in which case the ropelength is half of what we use here.

Suppose we are given an impenetrable tube of sufficient length so that the tube can be tied in a knotted conformation. There are two types of interactions between the tube's normal disks that restrict the possible conformations of the core curve. First, the tube cannot bend too quickly, a restriction on the curving of the core. Second, two distal (with respect to arclength) points in the core cannot be any closer than twice the radius of the tube, a restriction on the distance between pairs of points that are bounded away from the diagonal of $K \times K$. These intuitive observations are captured by the quantities below and the subsequent lemma.

Definition 2.2. For a $C^{2}$ knot $K$ with unit tangent map $T$, let $\operatorname{MinRad}(K)$ be the minimum radius of curvature of the points of the knot. The doubly critical self-distance is the minimum distance between pairs of points on the knot whose chord is perpendicular to the tangent vectors at the both of the points. In other words, let

$D C(K)=\{(x, y) \in K \times K: T(x) \perp \overline{x y} \perp T(y), x \neq y\}$,

where $\overline{x y}$ is the chord connecting $x$ and $y$. Define the doubly critical self-distance by

$$
\operatorname{dcs} d(K)=\min \{\|x-y\|:(x, y) \in D C(K)\},
$$


where $\|\cdot\|$ is the standard $\mathbb{R}^{3}$ norm. We call a pair $(x, y) \in D C(K)$ a doubly critical pair.

There is a fundamental relationship between $R(K)$, $\operatorname{MinRad}(K)$, and $d c s d(K)$.

Lemma 2.3. Suppose $K$ is a $C^{2}$ knot. Then $R(K)=$ $\min \left\{\operatorname{MinRad}(K), \frac{d c s d(K)}{2}\right\}$.

Proof: See [Litherland et al. 99].

After reworking the definition of MinRad, one can extend Lemma 2.3 to include $C^{1}$ curves. The following is taken from [Durumeric et al. 97]. For a $C^{0}$ function $f: \mathbb{R} \rightarrow \mathbb{R}^{3}$, define the dilation of $f$ by

$$
\operatorname{dil}(f)=\sup \left\{\frac{\|f(s)-f(t)\|}{|s-t|}: s, t \in \mathbb{R}, s \neq t\right\} .
$$

Note that if $K$ is a $C^{2}$ knot parameterized by arclength with unit tangent map $T$, then $\operatorname{MinRad}(K)=1 / \operatorname{dil}(T)$. Thus, the dilation gives a generalization for MinRad to knots that are $C^{1}$. Since MinRad and $1 / \operatorname{dil}(T)$ are equal for $C^{2}$ knots, we use MinRad to denote $1 / \operatorname{dil}(T)$ for all $C^{1}$ knots. In the case that a knot is $C^{1}$, but not $C^{1,1}$, $\operatorname{dil}(T)=\infty$, in which case MinRad is assumed to be 0 . In this paper, we are mainly interested in $C^{1,1}$ knots, in which case $\operatorname{dil}(T)$ is finite and MinRad is positive. Alternate, but equivalent, approaches for defining the ropelength of $C^{1,1}$ knots are explored in [Gonzalez and Maddocks 99, Cantarella et al. 02].

The dilation is similar to the distortion, another knot energy, defined in [Gromov 83] and studied in [O'Hara 92a, O'Hara 92b, Kusner and Sullivan 98]. We are using the dilation of the unit tangent map, not of a parameterization of a smooth knot as is the case in those papers. Our goal in using dilation is only to extend the definition of MinRad to $C^{1,1}$ knots.

Lemma 2.4. Suppose $K$ is a $C^{1}$ knot. Then $R(K)=$ $\min \left\{\operatorname{MinRad}(K), \frac{d \operatorname{csd}(K)}{2}\right\}$. Furthermore, if $K$ is $C^{1,1}$, then $R(K)>0$.

Proof: See [Cantarella et al. 02] or [Durumeric et al. 97].

It is standard protocol to use energy functions that are scale-invariant (since we are mainly interested in the "shape" of the optima) and have infinite barriers between knot types. Clearly, ropelength satisfies these properties.
In [Rawdon 98, Rawdon 00], the polygonal injectivity radius and polygonal ropelength functions were defined in the spirit of the characterizations in Lemmas 2.3 and 2.4 .

Suppose $P$ is an $n$-edge polygonal knot. We include a list of notation used throughout this paper.

- Let $\left\{v_{0}, \cdots, v_{n-1}\right\}$ be the vertices of $P$. For convenience, we implicitly take all subscripts modulo $n$.

- Let $\left\{e_{0}, \cdots, e_{n-1}\right\}$ be the edges of $P$, where $e_{i}$ is the edge connecting $v_{i}$ to $v_{i+1}$.

- Let $\left|e_{i}\right|$ be the length of the edge $e_{i}$.

- Let angle $\left(v_{i}\right)$ be the measure of the turning angle at $v_{i}$ (see Figure 1).

- Let $\theta_{\max }$ be the maximum of the turning angles.

- Given a knot $K$, polygonal or smooth, and $x \in K$, let $d_{x}: K \rightarrow \mathbb{R}$ be defined by $d_{x}(y)=\|x-y\|$.

- Let $\operatorname{arc}(x, y)$ be the minimum arc distance between $x$ and $y$.

Definition 2.5. For a vertex $v_{i}$ on an $n$-edge polygonal knot $P$, let

$$
\operatorname{Rad}\left(v_{i}\right)=\frac{\min \left\{\left|e_{i-1}\right|,\left|e_{i}\right|\right\}}{2 \tan \left(\frac{\operatorname{angle}\left(v_{i}\right)}{2}\right)}
$$

and

$$
\operatorname{MinRad}(P)=\min _{i=0, \cdots, n-1} \operatorname{Rad}\left(v_{i}\right) .
$$

Note that $\operatorname{Rad}\left(v_{i}\right)$ is the radius of a circular arc that can be inscribed at $v_{i}$ so that the arc is tangent to both edges adjacent to $v_{i}$ and the arc intersects the shorter adjacent edge at its midpoint (see Figure 1).

We call $y$ a turning point for $x$ if $d_{x}$ changes from increasing to decreasing or from decreasing to increasing at $y$. Let

$$
\begin{array}{r}
D C(P)=\{(x, y) \in P \times P: x \neq y \text { turning points of } \\
\left.d_{y} \text { and } d_{x}, \text { respectively }\right\} .
\end{array}
$$

Define the doubly critical self-distance of $P$ as

$$
\operatorname{dcsd}(P)=\min \{\|x-y\|:(x, y) \in D C(P)\} .
$$

We call a pair $(x, y) \in D C(P)$ a doubly critical pair, similar to the smooth case. 
Definition 2.6. For a polygonal knot $P$, let

$$
R(P)=\min \left\{\operatorname{MinRad}(P), \frac{d \operatorname{csd}(P)}{2}\right\}
$$

and

(polygonal injectivity radius)

$$
\rho(P)=\operatorname{Length}(P) / R(P) \text { (polygonal ropelength). }
$$

In [Rawdon 98, Rawdon 00], it was shown that for finer and finer inscribed polygonal approximations of a smooth knot $K$, the injectivity radius and ropelength of the polygons converge to the respective values of $K$.

\section{INSCRIBING SMOOTH KNOTS IN POLYGONS}

In Theorem 8.3, we show that polygonal ropelength minima converge to smooth ropelength minima. Thus, optimizing polygons do indeed show us the structure of the smooth optima. Alternate approaches for finding smooth energy optima using smooth curves are explored in [Kim and Kusner 93, Smutny and Maddocks 03]. In this section, we present the algorithm for inscribing smooth knots in polygonal knots and state the main result of this paper.

Proposition 3.1. For a given n-edge polygonal knot $P, a C^{1,1}$ curve $K$ can be inscribed in $P$ in such $a$ way that $\operatorname{MinRad}(K)=R(P)$ and the maximum of the minimum distance between a point on $P$ to $K$ is $\leq R(P)\left(\sec \left(\frac{\theta_{\max }}{2}\right)-1\right)$. Furthermore, there exists a bijection from $P$ to $K$ so that for each pair $x^{\prime} \in P$ and $x \in K$, we have $\left\|x-x^{\prime}\right\| \leq R(P)\left(\sec \left(\frac{\theta_{\max }}{2}\right)-1\right)$.

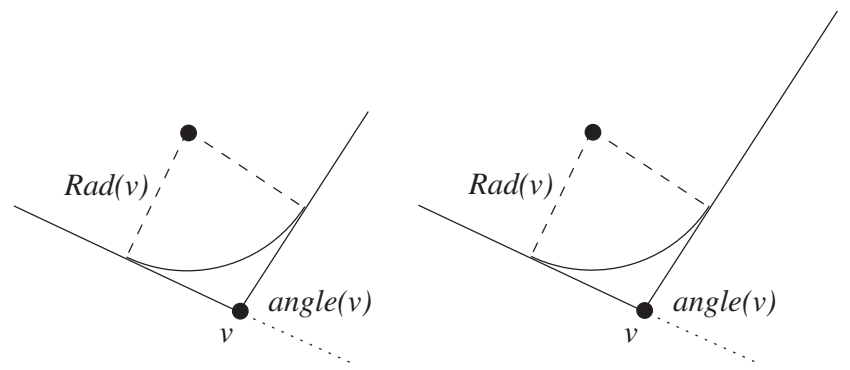

FIGURE 1. An arc of a circle of radius $\operatorname{Rad}(v)$ can be inscribed so that the arc is tangent at the midpoint of the shorter adjacent edge. On the left, the two adjacent edges have identical length, so the arc intersects both edges tangentially at the midpoints. On the right, the arc intersects the longer edge short of the midpoint.
Proof: Recall from Definition 2.5 that an arc $\alpha_{i}$ of a circle of radius $\operatorname{Rad}\left(v_{i}\right)$ can be inscribed at $v_{i}$ such that $\alpha_{i}$ is tangent to $e_{i-1}$ and $e_{i}$ and intersects the shorter adjacent edge at the midpoint. Since $R(P) \leq \operatorname{MinRad}(P) \leq$ $\operatorname{Rad}\left(v_{i}\right)$, an arc of radius $R(P)$ can be inscribed at $v_{i}$ tangent to $e_{i-1}$ and $e_{i}$. The points of intersection between $\alpha_{i}$ and the edges will be no further away from $v_{i}$ than the midpoints of the edges (see Figure 2).

Let $K$ be the result of inscribing arcs of radius $R(P)$ in $P$ and removing the bypassed corners. Since there is no overlapping of adjacent inscribed arcs (although they could meet in a $C^{1}$ fashion at a midpoint), $K$ is well defined as a (possibly self-intersecting) closed curve. The curve $K$ is the union of arcs of radius $R(P)$ and (possibly) straight line segments meeting tangentially, so $K$ is $C^{1}$ and piecewise $C^{2}$. Thus, $K$ lies in the category of $C^{1,1}$ curves. By this construction, we have that $\operatorname{MinRad}(K)=R(P)$.

For each $x$ on the inscribed curve $K$, we define a unique point $x^{\prime}$ on $P$. If $x$ is on a line segment, let $x^{\prime}=x$. Otherwise, $x$ lies on an arc, say $\alpha_{i}$, whose center is $C_{i}$. Let $x^{\prime}$ be the intersection of the ray $\overrightarrow{C_{i} x}$ with $e_{i-1} \cup e_{i}$ (see Figure 2). Simple trigonometric calculations show that

$$
\left\|x-x^{\prime}\right\| \leq R(P)\left(\sec \left(\frac{\theta_{\max }}{2}\right)-1\right) .
$$

The inscribed curve $K$ is the object of study in this paper. Our main result is that we can find an explicit bound for $R(K)$ in terms of $R(P)$ and $\theta_{\max }$, the maximum turning angle of $P$. For polygons with sufficiently large $R(P)$ and sufficiently small $\theta_{\max }$, we can guarantee that $R(K)>0$, so $K$ is truly a knot; furthermore, when $\theta_{\text {max }} \lesssim 96^{\circ}$, we show that $P$ and $K$ have the same knot type.

Note that at a vertex $v_{i}$, one could inscribe a circular arc of radius $r \leq \operatorname{Rad}\left(v_{i}\right)$ and still have a well-defined smooth knot. Larger values of $r$ increase the distance between $x$ and $x^{\prime}$. We want $P$ and $K$ to be close to each other, so $r$ should be small to minimize this error. However, $\operatorname{MinRad}(K)$ is the minimum arc radius used in inscribing $K$ in $P$, which we want as large as possible. We choose $r=R(P)$ to minimize the distance between $x$ and $x^{\prime}$ without adversely affecting the injectivity radius of $K$ (which we want to be close to $R(P)$ ).

The following is the main result of this paper.

Theorem 3.2. Suppose $P$ is a polygonal knot. Then there exists a $C^{1,1}$ knot $K$ inscribed in $P$ such that

$$
R(P)-R(P)\left(\sec \left(\frac{\theta_{\max }}{2}\right)-1\right) \leq R(K) \leq R(P) .
$$




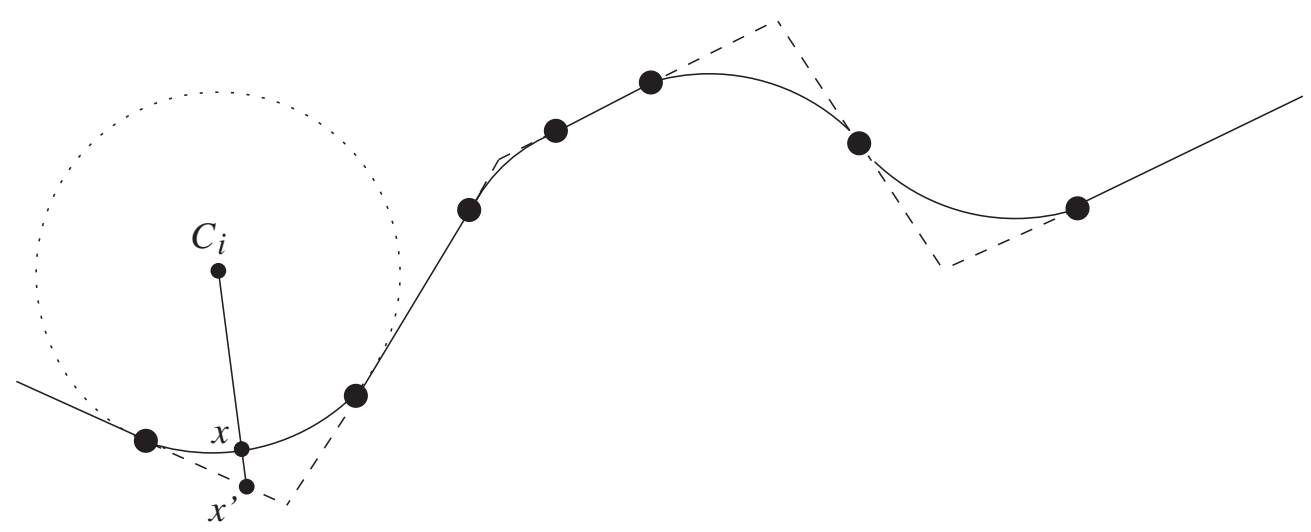

FIGURE 2. A smooth knot is inscribed in a portion of a polygon.

The proof of Theorem 3.2 is in Section 6. The proof relies on new characterization theorems for both $R(K)$ and $R(P)$.

\section{CHARACTERIZATION THEOREMS FOR $R(K)$}

In this section, we prove alternate characterizations for the injectivity radius of smooth knots. These lemmas are similar to the characterizations in Section 2. The goal is to replace $d c s d$ with a different term which lets us better understand the relationship between the polygon and its inscribed smooth curve.

Recall that $d c s d$ is the minimum distance between pairs of points in $D C(K)$, the set of doubly critical pairs. The set $D C(K)$ is a subset of $K \times K$ bounded away from the diagonal. We will replace $D C(K)$ with other subsets of $K \times K$ defined in terms of total curvature and arclength.

We begin by reviewing an alternate characterization of $R(P)$ from [Rawdon 98, Rawdon 00] which utilizes the total curvature between two points on a polygon. The main theorem relies on a similar result for smooth knots which appears at the end of this section.

Definition 4.1. Let $P$ be a polygonal knot and $p \neq q \in P$. For each of the two arcs connecting $p$ and $q$, sum the turning angles between $p$ and $q$ (and in the case that $p$ and/or $q$ is a vertex, including angle $(p)$ and/or angle $(q))$. Let $t c(p, q)$ be the smaller of the two quantities.

Lemma 4.2. Let $T C(P)=\{(p, q) \in P \times P: t c(p, q) \geq \pi\}$. Then

$$
R(P)=\min \left\{\operatorname{MinRad}(P), \min _{(p, q) \in T C(P)} \frac{\|p-q\|}{2}\right\} .
$$

Proof: See [Rawdon 00].

We want to extend this result to $C^{1,1}$ knots. However, we must be careful in defining the total curvature for $C^{1,1}$ knots. For a $C^{2}$ knot $K$, the total curvature of $K$ is simply the integral of the scalar curvature function. In [Milnor 50], the definition of total curvature is extended to incorporate all continuous curves. Milnor defines the total curvature of a $C^{0}$ arc to be the supremum, over all inscribed polygons, of the sum of the turning angles of the polygon.

Definition 4.3. For a pair of points $x, y$ on a $C^{1,1}$ knot $K$, let $t c(x, y)$ be the minimum total curvature between the two points in the sense of Milnor.

While our notion of total curvature (Definition 4.1) agrees with Milnor's notion on $C^{1,1}$ arcs and polygonal arcs with nonvertex endpoints, our definition differs slightly for polygonal arcs with a vertex endpoint. When an endpoint of a polygonal arc is a vertex, we include that vertex angle in measuring the total curvature of the arc. This does not pose any problems in this work.

The following three lemmas and theorem simplify the proof of the new characterization. We first show that a certain amount of total curvature is necessary to achieve a doubly critical pair.

Lemma 4.4. For points $x$ and $y$ on a $C^{1,1}$ knot $K$, if $t c(x, y)<\pi$, then $(x, y)$ is not a doubly critical pair.

Proof: If $(x, y)$ is a doubly critical pair and $t c(x, y)<\pi$, then the union of the arc on which $t c(x, y)<\pi$ with the chord $\overline{x y}$ forms a closed curve with total curvature (in 
the sense of Milnor) less than $2 \pi$, which is a contradiction [Milnor 50].

Next, we bound the total curvature between points on a $C^{1,1}$ knot in terms of arclength and MinRad.

Lemma 4.5. For a $C^{1,1}$ knot $K$, let $\operatorname{MaxCurv}(K)=$ $\operatorname{dil}(T)=1 / \operatorname{MinRad}(K)$. Then

$$
\begin{gathered}
\angle(T(x), T(y)) \leq \operatorname{Max} C u r v(K) \cdot \operatorname{arc}(x, y), \\
t c(x, y) \leq M a x C u r v(K) \cdot \operatorname{arc}(x, y), \text { and } \\
t c(K) \leq \operatorname{Max} C u r v(K) \cdot \operatorname{Length}(K),
\end{gathered}
$$

where $\angle(T(x), T(y))$ is the angle between the tangent vectors $T(x)$ and $T(y)$.

Proof: See [Durumeric et al. 97].

We use Schur's Theorem, which compares the chord distance of a space curve with that of a planar reference curve, to bound the distance between pairs of points in subsets of $K \times K$. This form of Schur's Theorem appears in [Chern 67].

\section{Lemma 4.6. (Schur's Theorem for Piecewise Smooth}

Curves.) Let $C$ and $C^{*}$ be two piecewise smooth curves of the same length, such that $C$, together with the chord connecting its endpoints, forms a simple convex plane curve. Let $s$ be the arclength parameter for $C$ and $C^{*}$. Let $\kappa(s)$ be the curvature of $C$ at a regular point, a $(s)$ the angle between the oriented tangents at a vertex, and denote corresponding quantities for $C^{*}$ by the same notations with asterisks. Let $d$ and $d^{*}$ be the distances between the endpoints of $C$ and $C^{*}$, respectively. Then, if

$$
\kappa^{*}(s) \leq \kappa(s) \text { and } a^{*}(s) \leq a(s)
$$

we have $d^{*} \geq d$. In other words, less curving implies greater endpoint distances, so long as the reference curve is planar.

The following characterization is used in the proof of the next theorem.

Lemma 4.7. Let $A(K)=\{(x, y) \in K \times K: \operatorname{arc}(x, y) \geq$ $\pi \operatorname{MinRad}(K)\}$. For a $C^{1,1}$ knot $K$,

$$
R(K)=\min \left\{\operatorname{MinRad}(K), \min _{(x, y) \in A(K)} \frac{\|x-y\|}{2}\right\} .
$$

Proof: If $\operatorname{arc}(x, y)<\pi M i n R a d(K)$, then $t c(x, y)<$ $\pi \operatorname{MinRad}(K) \cdot \operatorname{Max} \operatorname{Curv}(K)=\pi$ and Lemma 4.4 says that $(x, y)$ cannot be a doubly critical pair. Thus,

$$
\min _{(x, y) \in A(K)}\|x-y\| \leq d \operatorname{csd}(K) .
$$

If $\operatorname{MinRad}(K) \leq \min _{(x, y) \in A(K)} \frac{\|x-y\|}{2}$, then $R(K)=$ $\operatorname{MinRad}(K) \leq d \operatorname{csd}(K) / 2$ and the result follows.

Otherwise, $\min _{(x, y) \in A_{K}} \frac{\|x-y\|}{2}<\operatorname{MinRad}(K)$. Suppose $\operatorname{arc}(x, y)=\pi \operatorname{MinR} \operatorname{Rad}(K)$. By comparing the arc between $x$ and $y$ with a semicircle of radius $r=$ $\operatorname{MinRad}(K)$ and applying Schur's Theorem, we get that $\|x-y\| \geq 2 \operatorname{MinRad}(K)$. Since $\frac{\|x-y\|}{2} \geq \operatorname{MinRad}(K)$ on the boundary of $A(K)$, the minimum distance between pairs of points in $A(K)$ is attained on an open set and, thus, must be realized at a doubly critical pair. In other words, $R(K)=\frac{d \operatorname{csd}(K)}{2}=\min _{(x, y) \in A(K)} \frac{\|x-y\|}{2}$.

We now prove the $C^{1,1}$ version of Lemma 4.2 .

Theorem 4.8. Let $T C(K)=\{(x, y) \in K \times K: t c(x, y) \geq$ $\pi\}$. For a $C^{1,1}$ knot $K$,

$$
R(K)=\min \left\{\operatorname{MinRad}(K), \min _{(x, y) \in T C(K)} \frac{\|x-y\|}{2}\right\} .
$$

Proof: If $t c(x, y)<\pi$, then $(x, y)$ cannot be a doubly critical pair by Lemma 4.4. Thus,

$$
\min _{(x, y) \in T C(K)}\|x-y\| \leq d \operatorname{csd}(K) .
$$

If $\operatorname{MinRad}(K) \leq \min _{(x, y) \in T C(K)} \frac{\|x-y\|}{2}$, then $R(K)=$ $\operatorname{MinRad}(K) \leq \operatorname{dcsd}(K) / 2$ and the result holds.

Otherwise, $\min _{(x, y) \in T C(K)} \frac{\|x-y\|}{2}<\operatorname{MinRad}(K)$. Since $T C(K) \subseteq A(K)$, we have $\min _{(x, y) \in A(K)} \frac{\|x-y\|}{2} \leq$ $\min _{(x, y) \in T C(K)} \frac{\|x-y\|}{2}<\operatorname{MinRad}(K)$. Thus, $\operatorname{dcsd}(K)=$ $\min _{(x, y) \in A(K)}\|x-y\| \leq \min _{(x, y) \in T C(K)}\|x-y\| \leq$ $\operatorname{dcsd}(K)$.

We can now describe the basic structure of the proof of Theorem 3.2. On the inscribed knot $K, \operatorname{Min} \operatorname{Rad}(K)=$ $R(P)$. So if $\operatorname{MinRad}(K) \leq \operatorname{dcsd}(K) / 2$, then $R(K)=$ $R(P)$. If $d \operatorname{csd}(K) / 2<\operatorname{MinRad}(K)$, we need to show that $d \operatorname{csd}(K) \approx d \operatorname{csd}(P)$. If a pair $(x, y)$ realizes $d c s d(K)$ and $t c\left(x^{\prime}, y^{\prime}\right) \geq \pi$ on $P$, then the theorem follows immediately.

While the total curvature between $x, y \in K$ is close to the total curvature of the corresponding points $x^{\prime}, y^{\prime} \in P$, they need not be identical. The total curvature of a 
polygonal arc is the sum of the turning angles at the vertices on the arc (including the first and/or last point if it is a vertex). The total curvature between $x^{\prime}, y^{\prime} \in P$ is the minimum of the total curvature along the two arcs joining the points. Thus, total curvature accumulates along an arc of a polygon as a jump function at the vertices. However, on a smooth curve, total curvature accumulates continuously as the tangent vector turns.

In fact, on a polygon $P$ with inscribed smooth knot $K$, there exist $x_{0}, y_{0} \in K$ such that $t c\left(x_{0}, y_{0}\right)<t c\left(x_{0}^{\prime}, y_{0}^{\prime}\right)$ and $x_{1}, y_{1} \in K$ such that $t c\left(x_{1}^{\prime}, y_{1}^{\prime}\right)<t c\left(x_{1}, y_{1}\right)$. This behavior occurs near vertices. For example, suppose $f$ is an arclength parameterization of $K$ such that $f(a)^{\prime}$ and $f(b)^{\prime}$ (the points on $P$ associated to $f(a)$ and $\left.f(b)\right)$ are vertices with $a<b$ and $t c(f(a), f(b))$ realized on the arc from $f(a)$ to $f(b)$. The total curvature $\operatorname{tc}(f(a), f(b))<$ $t c\left(f(a)^{\prime}, f(b)^{\prime}\right)$ since the smooth knot has not completed the full turning of the circular arc inscribed at the two vertices. On the other hand, for small $\epsilon>0$, the total curvature $t c(f(a+\epsilon), f(b-\epsilon))>t c\left(f(a+\epsilon)^{\prime}, f(b-\epsilon)^{\prime}\right)$ since the smooth curve has accumulated some of the total curvature from the two circular arcs that has not yet registered as total curvature on the polygon arc.

The critical case for the proof of Theorem 3.2 occurs when $\operatorname{dcsd}(K) / 2<\operatorname{MinRad}(K)$ and $R(K)$ is realized at a pair $(x, y)$ with $t c(x, y) \geq \pi$ but $t c\left(x^{\prime}, y^{\prime}\right)<\pi$. In such a situation, one would expect the distance from $x^{\prime}$ to $y^{\prime}$ to be close to $2 \operatorname{MinRad}(P)$. One can show that the vertices preceding $x^{\prime}$ and following $y^{\prime}$, say $v_{0}$ and $v_{k}$, have total curvature at least $\pi$. Thus,

$$
\begin{aligned}
R(K)= & \frac{\|x-y\|}{2} \\
\geq \frac{\left\|v_{0}-v_{k}\right\|}{2} & -(\text { two edge lengths }) \\
& \quad-R(P)\left(\sec \left(\frac{\theta_{\max }}{2}\right)-1\right) \\
\geq R(P)-(\text { two edge lengths }) & \quad-R(P)\left(\sec \left(\frac{\theta_{\max }}{2}\right)-1\right) .
\end{aligned}
$$

This argument bounds $R(K)$ below, but the error of two edge lengths is not necessary. We can improve the error by proving a new characterization of $R(P)$.

\section{CHARACTERIZATION THEOREMS FOR $R(P)$}

In this section, we use a different definition of the total curvature on $P$ to prove a characterization of $R(P)$. This is the last piece needed for the proof of the main theorem. We begin by defining $t c^{*}$.
Definition 5.1. For a polygonal knot $P$ with $K$ inscribed via Proposition 3.1, let $t c^{*}\left(x^{\prime}, y^{\prime}\right)=t c(x, y)$. In other words, the new measure of the total curvature between two points on $P$ is the total curvature between the corresponding points of $K$.

In the proofs of the last section, we used the fact that when the total curvature between two points is exactly $\pi$, the distance between the points is at least 2 MinRad. Thus, when $R(K)$ is realized by $d c s d$, the minimum distance over $T C(K)$ and $A(K)$ is realized at a doubly critical pair. The following lemma establishes this result for polygons using $t c^{*}$.

Lemma 5.2. Suppose $P$ is a polygonal knot with inscribed smooth knot $K$ via Proposition 3.1. If $t c^{*}\left(x^{\prime}, y^{\prime}\right)=\pi$, then $\left\|x^{\prime}-y^{\prime}\right\| \geq 2 R(P)$.

Proof: Let $x^{\prime}, y^{\prime} \in P$ such that $t c^{*}\left(x^{\prime}, y^{\prime}\right)=\pi$. Consider an arc of $P$ which realizes $t c^{*}\left(x^{\prime}, y^{\prime}\right)=\pi$ and the corresponding $\operatorname{arc}$ on $K$. With the intent of applying Schur's Theorem, we create planar reference curves, called $C_{P}$ and $C_{K}$, for the arcs of $P$ and $K$. The arc of $P$ contains a set of vertices, say $\left\{v_{1}, \cdots, v_{k}\right\}$ (do not include $x^{\prime}$ or $y^{\prime}$ in this list), connecting a set of edges, $\left\{e_{1}, \cdots, e_{k-1}\right\}$. Let $e_{0}$ be the line segment from $x^{\prime}$ to $v_{1}$ and $e_{k}$ the line segment from $v_{k}$ to $y^{\prime}$. Construct a planar oriented arc $C_{P}$ as follows:

- Let $p_{0}=(0,0)$.

- Let $p_{1}$ be the point $\left(\left\|x^{\prime}-v_{1}\right\|, 0\right)$.

- Let $p_{2}$ be the point lying above the $x$-axis such that $\overrightarrow{p_{1} p_{2}}$, makes a turning angle (counterclockwise) of $\operatorname{angle}\left(v_{1}\right)$ with the vector $\overrightarrow{p_{0} p_{1}}$ and $\left\|p_{1}-p_{2}\right\|=\| v_{1}-$ $v_{2} \|$.

- Let $p_{i}$ be the point such that $\overrightarrow{p_{i-1} p_{i}}$, makes a turning angle (counterclockwise) of angle $\left(v_{i}\right)$ with the vector $\overrightarrow{p_{i-2} p_{i-1}}$ and $\left\|p_{i-1}-p_{i}\right\|=\left\|v_{i-1}-v_{i}\right\|$.

- Let $p_{k+1}$ be the point such that $\overrightarrow{p_{k} p_{k+1}}$, makes a turning angle (counterclockwise) of angle $\left(v_{k}\right)$ with the vector $\overrightarrow{p_{k-1} p_{k}}$ and $\left\|p_{k}-p_{k+1}\right\|=\left\|v_{k}-y^{\prime}\right\|$.

Then $C_{P}$ has identical angles and edge lengths as the arc of $P$. We construct $C_{K}$ similarly so that it is based at the origin with the initial tangent pointing in the direction 
$(1,0)$ and so that the curvature of $C_{K}$ is identical to the arc of $K$, with the turning of the tangent vector occurring counterclockwise in the plane. Then $C_{K}$ lies in the region of the plane with $y \geq 0$.

We now show that the endpoint distance of $C_{K}$ is at least $2 R(P)$. Let $S$ be the semicircle of radius $R(P)$ with center $(0, R(P))$ lying in the first-quadrant. The semicircle $S$ has an endpoint distance of $2 R(P)$ and the tangents of $S$ all have a non-negative $y$-component. Since the arc $C_{K}$ is just a semicircle with (possibly) some straight line segments inserted so that $C_{K}$ is $C^{1,1}$, the tangents of $C_{K}$ also all have a non-negative $y$-component. Furthermore, the endpoint distance of $C_{K}$ is at least as large as the $y$-coordinate difference between the endpoints. Since all of the tangents of $C_{K}$ have a non-negative $y$-component, the $y$-coordinate difference of $C_{K}$ is at least as large as the $y$-coordinate difference of $S$, which is $2 R(P)$. Thus, the endpoint distance of $C_{K}$ is at least $2 R(P)$.

By the construction of $C_{K}$, one can translate and rotate $C_{P}$ such that $C_{K}$ is inscribed in $C_{P}$ just as if we were inscribing a smooth curve in $C_{P}$ via the algorithm in Proposition 3.1. Translate and rotate $C_{P}$ so that $C_{K}$ is inscribed in $C_{P}$. Then the initial point of $C_{P}$ must lie on the nonpositive portion of the $y$-axis. Furthermore, the final point of $C_{K}$ must have its tangent in the direction $(-1,0)$ (parallel to the $x$-axis) on the line $y=M \geq 2 R(P)$. By the construction of the inscribed smooth curve $C_{K}$, the final point of $C_{P}$ must lie on a vertical line containing the final point of $C_{K}$ and lie above $y=M$. Thus,

endpoint distance of $C_{P} \geq y$-coordinate difference of endpoints of $C_{P}$ $\geq y$-coordinate difference of endpoints of $C_{K}$ $\geq 2 R(P)$.

Thus, by Schur's Theorem, the endpoint distance of the original arc of $P$ must be at least as large as the endpoint distance of $C_{P}$, i.e., $\left\|x^{\prime}-y^{\prime}\right\| \geq 2 R(P)$.

We now prove the characterization theorem for $R(P)$.

Theorem 5.3. Let $B(P)=\left\{\left(x^{\prime}, y^{\prime}\right) \in P \times P\right.$ : $t c^{*}\left(x^{\prime}, y^{\prime}\right) \geq \pi$ or $\left.t c\left(x^{\prime}, y^{\prime}\right) \geq \pi\right\}$. For a polygonal knot $P$,

$$
R(P)=\min \left\{\operatorname{MinRad}(P), \min _{\left(x^{\prime}, y^{\prime}\right) \in B(P)} \frac{\left\|x^{\prime}-y^{\prime}\right\|}{2}\right\} .
$$

Proof: Notice that since $T C(P) \subseteq B(P)$,

$\min _{\left(x^{\prime}, y^{\prime}\right) \in B(P)}\left\|x^{\prime}-y^{\prime}\right\| \leq \min _{\left(x^{\prime}, y^{\prime}\right) \in T C(P)}\left\|x^{\prime}-y^{\prime}\right\| \leq d \operatorname{csd}(P)$.

If $\operatorname{MinRad}(P) \leq \min _{\left(x^{\prime}, y^{\prime}\right) \in B(P)} \frac{\left\|x^{\prime}-y^{\prime}\right\|}{2}$, then $R(P)=$ $\operatorname{MinRad}(P) \leq d \operatorname{csd}(P) / 2$ and the result holds.

Suppose $\min _{\left(x^{\prime}, y^{\prime}\right) \in B(P)} \frac{\left\|x^{\prime}-y^{\prime}\right\|}{2}<\operatorname{MinRad}(P)$. Now $T C(P)$ is closed by [Rawdon 00] and $T C^{*}(P)=\{(p, q) \in$ $\left.P \times P: t c^{*}(p, q) \geq \pi\right\}$ is also closed. Thus, $B(P)=$ $T C(P) \cup T C^{*}(P)$ is closed. The boundary of $B(P)$ is contained in the union of the boundaries of $T C(P)$ and $T C^{*}(P)$. On $\partial(T C(P)),\left\|x^{\prime}-y^{\prime}\right\| \geq 2 \operatorname{MinRad}(P)$ by [Rawdon 00] and on $\partial\left(T C^{*}(P)\right),\left\|x^{\prime}-y^{\prime}\right\| \geq 2 R(P)$ by the Lemma 5.2. If $\min _{\left(x^{\prime}, y^{\prime}\right) \in B(P)} \frac{\left\|x^{\prime}-y^{\prime}\right\|}{2}<R(P)$, then the minimum is realized on the interior of $B(P)$, in which case the minimum must be realized at a doubly critical pair. But this contradicts the assumption that $\min _{\left(x^{\prime}, y^{\prime}\right) \in B(P)} \frac{\left\|x^{\prime}-y^{\prime}\right\|}{2}<R(P)$. Thus, if $\min _{\left(x^{\prime}, y^{\prime}\right) \in B(P)} \frac{\left\|x^{\prime}-y^{\prime}\right\|}{2}<\operatorname{MinRad}(P)$, then

$$
\min _{\left(x^{\prime}, y^{\prime}\right) \in B(P)} \frac{\left\|x^{\prime}-y^{\prime}\right\|}{2}=\frac{d c s d(P)}{2}=R(P) .
$$

We have the tools to prove the main result of this paper.

\section{THE MAIN THEOREM}

We use the characterizations from the last two sections to bound the injectivity radius of the inscribed smooth knot. We also show that when the maximum turning angle $\theta_{\max }$ is sufficiently small, the knots $P$ and inscribed $K$ have the same knot type.

We begin with the proof of Theorem 3.2.

Proof of Theorem 3.2: Let $K$ be inscribed in $P$ via the algorithm of Proposition 3.1. For the upper bound, note that $\operatorname{MinRad}(K)=R(P)$, so $R(K) \leq R(P)$.

The lower bound splits into two cases. First, note that if $\operatorname{MinRad}(K) \leq d \operatorname{csd}(K) / 2$, then $R(K)=$ $\operatorname{MinRad}(K)=R(P)$.

For the second case, we assume $\operatorname{dcs} d(K) / 2<$ $\operatorname{MinRad}(K)$. Thus, $R(K)=\operatorname{dcsd}(K) / 2=$ $\min _{(x, y) \in T C(K)} \frac{\|x-y\|}{2}$ by Theorem 4.8. Let $\left(x_{0}, y_{0}\right)$ be a pair in $T C(K)$ realizing the minimum distance, that is $\left(x_{0}, y_{0}\right) \in T C(K)$ with $R(K)=\frac{\left\|x_{0}-y_{0}\right\|}{2}$. Then $t c^{*}\left(x_{0}^{\prime}, y_{0}^{\prime}\right)=t c\left(x_{0}, y_{0}\right) \geq \pi$ and $\left(x_{0}^{\prime}, y_{0}^{\prime}\right) \in B(P)$. Thus,

$$
\left\|x_{0}^{\prime}-y_{0}^{\prime}\right\|-2 R(P)\left(\sec \left(\frac{\theta_{\max }}{2}\right)-1\right) \leq\left\|x_{0}-y_{0}\right\|
$$$$
=2 R(K)(6-1)
$$ 
by Proposition 3.1. Since $\left(x_{0}^{\prime}, y_{0}^{\prime}\right) \in B(P)$,

$$
R(P) \leq \frac{\left\|x_{0}^{\prime}-y_{0}^{\prime}\right\|}{2}
$$

Dividing (6-1) by two and combining with (6-2) yields

$$
R(P)-R(P)\left(\sec \left(\frac{\theta_{\max }}{2}\right)-1\right) \leq R(K) .
$$

If $P$ is ropelength minimized and "looks" fairly smooth, one would expect that $P$ and $K$ have the same knot type. We show, in fact, that $P$ and $K$ have the same knot type when $\theta_{\max } \lesssim 96^{\circ}$. In the subsequent sections, we are mainly interested in "thick" polygons with many edges, in which case $\theta_{\max }$ will be close to 0 .

Theorem 6.1. If $P$ is a polygonal knot with $K$ inscribed in $P$ as in Proposition 3.1 and $\theta_{\max }<2 \operatorname{arcsec}\left(\frac{3}{2}\right)$, then $P$ and $K$ have the same knot type.

Proof: At each vertex of $P$, we have inscribed an arc to create $K$. This construction creates dented triangles between $P$ and $K$ (see Figure 3 ). If no two dented triangles intersect, then the knot types of $P$ and $K$ are identical. We show that dented triangles are disjoint when $\theta_{\max }<2 \operatorname{arcsec}\left(\frac{3}{2}\right)$ by proving that $K$ is "thick" enough so that the polygon $P$, and thus, the dented triangles, lie inside the tube of radius $R(K)$ about $K$.

By Theorem 3.2, $R(K)>R(P)\left(2-\sec \left(\frac{\theta_{\max }}{2}\right)\right)$. Thus, for all $r<R(K)$ and each pair $x \neq y \in K$, the normal disks of radius $r$ centered at $x$ and $y$ are disjoint. By Proposition 3.1, for all $x \in K$ and corresponding $x^{\prime} \in P$, we have $\left\|x-x^{\prime}\right\| \leq R(P)\left(\sec \left(\frac{\theta_{\max }}{2}\right)-1\right)$. Since $\theta_{\max }<2 \operatorname{arcsec}\left(\frac{3}{2}\right), R(K)>\left\|x-x^{\prime}\right\|$. Thus, when $r=R(P)\left(\sec \left(\frac{\theta_{\max }}{2}\right)-1\right)$, each $x^{\prime}$, and thus each point of each dented triangle, lies on a unique normal disk. Since these normal disks do not intersect, the dented triangles do not intersect and $P$ and $K$ have the same knot type.

We have now shown that we can inscribe a smooth knot $K$ in a sufficiently thick polygon $P$ so that $R(P) \approx$ $R(K)$ and $P$ and $K$ have the same knot type. In the following section, we use these results to compute upper bounds on the smooth ropelength of different knot types.

\section{COMPUTATION OF UPPER BOUNDS ON SMOOTH ROPELENGTH}

We can determine true upper bounds for the ropelength of smooth knot types. Let $P$ be a ropelength minimized

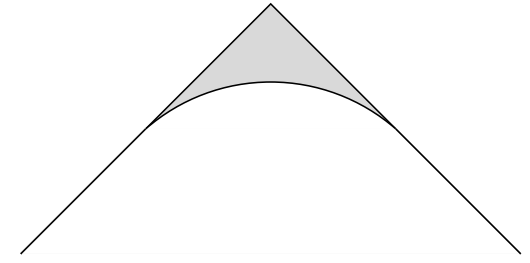

FIGURE 3. The shaded portion between the polygon and the inscribed arc is the dented triangle from the proof of Theorem 6.1.

polygon of a given knot type $\mathcal{K}$. We apply Theorem 3.2 to obtain a lower bound for the injectivity radius of the inscribed smooth knot $K$. If $\theta_{\max }$ is sufficiently small (which it will be if $P$ is sufficiently thick and has enough edges), Theorem 6.1 says that $K$ also has knot type $\mathcal{K}$. The minimal smooth ropelength within $\mathcal{K}$ is at most the ropelength of $K$ since $K$ is one conformation within the knot type. We are not claiming that $K$ is ropelength minimal or even that we know the exact ropelength of the inscribed knot $K$. Rather, by bounding $\rho(K)$ from above, we know that a smooth ropelength minimum within $\mathcal{K}$ must have ropelength smaller than our computed upper bound. The inscribed smooth knot has shorter arclength than the polygon (we bypassed some corners); the computations reflect the true length of the smooth inscribed knot.

We computed upper bounds for the smooth ropelength of all prime knots through nine crossings. The starting conformations were provided by Pierański, who has computed minimizing polygonal conformations for knots with many edges [Katritch et al. 96, Katritch et al. 97, Pierański 98] using the efficient SONO algorithm [Pierański 97, Pierański 98]. While the SONO algorithm does not explicitly minimize the polygonal ropelength discussed here, ropelength minimizing conformations tend to be very close to SONO minimized polygons (see [Stasiak et al. 98] and [Millett and Rawdon 03] for a comparison).

We use a deterministic ropelength minimizing algorithm to insure (up to the maximum reliability of double calculations) that each knot is vertex-critical, that is no small perturbation in the $x, y$, or $z$ directions reduces the ropelength. We believe that each conformation is near a global minimum, but there is currently no known criteria for determining whether a knot is at a global minimum. To check for vertex-criticality, we search the tangent space for small perturbations that reduce the ropelength. The tangent space of a polygon is spanned by the $3 n$ perturbations determined by moving each ver- 


\begin{tabular}{|c|c|c|c|c|c|c|c|}
\hline Inot & Edges & oly $\rho$ & Bound & Knot & Edges & Poly $\rho$ & Bour \\
\hline $3_{1}$ & 160 & 32.80 & 32.90 & $9_{8}$ & 400 & 80.78 & 81.1 \\
\hline 3 & 54 & 32.76 & 32.77 & $\overline{9_{9}}$ & 392 & 80.43 & 80.85 \\
\hline $4_{1}$ & 08 & 42.19 & 42.33 & $9_{10}$ & 392 & 9.97 & .33 \\
\hline 5 & 232 & 47.30 & 47.51 & $9_{11}$ & 400 & 81.61 & 81.98 \\
\hline $5_{2}$ & 240 & 49.59 & 49.73 & $9_{12}$ & 400 & 0.35 & 0.7 \\
\hline $66_{1}$ & 280 & 56.85 & 57.11 & $9_{13}$ & 400 & 80.96 & 81.3 \\
\hline $6_{2}$ & 280 & 57.19 & 57.44 & $9_{14}$ & 400 & 80.32 & 80.7 \\
\hline $6_{3}$ & 288 & 58.28 & 58.48 & $9_{15}$ & 408 & 82.32 & 82.70 \\
\hline $7_{1}$ & 304 & 61.66 & 61.89 & $9_{16}$ & 400 & 80.33 & 80.67 \\
\hline $7_{2}$ & 320 & 65.08 & 65.36 & $9_{17}$ & 400 & 81.46 & 81.90 \\
\hline 73 & 312 & .06 & 64.35 & 9 & 400 & 82.24 & 82.68 \\
\hline $7_{4}$ & 320 & 5.33 & 65.63 & 19 & 08 & 82.36 & 2.7 \\
\hline $7_{5}$ & 320 & 65.41 & 65.70 & ${ }^{2} 20$ & 424 & 86.73 & 7.5 \\
\hline $7_{6}$ & 328 & 65.91 & 66.17 & $9_{21}$ & 400 & 81.31 & 81.64 \\
\hline $7_{7}$ & 328 & 65.81 & 66.09 & $9_{22}$ & 400 & 81.23 & 81.60 \\
\hline 81 & 352 & 71.22 & 71.4 & $9_{23}$ & 400 & 81.47 & 81.8 \\
\hline $8_{2}$ & 352 & 71.5 & 71 & $9_{24}$ & 00 & 81.17 & 81.5 \\
\hline 83 & 352 & 71.27 & 71.56 & $9_{25}$ & $\overline{00}$ & 81.37 & 81.85 \\
\hline 84 & 552 & 72.15 & 72.41 & $9_{26}$ & 0 & 81.56 & 81.94 \\
\hline 85 & 60 & 72.36 & 72.70 & $9_{27}$ & 08 & 82.72 & 83.21 \\
\hline $8_{6}$ & 360 & 72.60 & 72.93 & $y_{28}$ & 400 & 81.87 & 82.25 \\
\hline 87 & 360 & 72.39 & 72.63 & $9_{29}$ & 400 & 82.75 & 83.45 \\
\hline 88 & 360 & 73.54 & 73 & $9_{30}$ & 08 & 82.09 & 82.46 \\
\hline 89 & 360 & 72.63 & 72.96 & $9_{3}$ & 400 & 81.84 & 82.22 \\
\hline 810 & 360 & 73.58 & 73.86 & $99_{32}$ & 00 & 81.95 & 82.34 \\
\hline 811 & 376 & 76.35 & 76.70 & $9_{33}$ & 08 & 3.03 & 83.37 \\
\hline 812 & 368 & 74.27 & 74.61 & 934 & 408 & 2.62 & 82.99 \\
\hline 813 & 360 & 73 & 73.29 & 9 & 400 & 50 & 80. \\
\hline 814 & 368 & 55 & 74 . & & 400 & 1.21 & 81.57 \\
\hline 815 & 376 & 4.50 & 74.82 & $\overline{9_{37}}$ & 00 & 81.73 & 82.10 \\
\hline 816 & 368 & 75.13 & 75.47 & $9_{38}$ & 408 & 82.06 & 82.43 \\
\hline 817 & 368 & 74.74 & 75.08 & 9 & 416 & 85.14 & 85.55 \\
\hline 818 & 368 & 75.12 & 75.44 & $9_{40}$ & 400 & 82.26 & 82.67 \\
\hline 819 & 304 & 61.16 & 61.35 & $9_{41}$ & 400 & 81.73 & 82.11 \\
\hline $8_{20}$ & 312 & 63.74 & 64 & $9_{42}$ & 14 & 9.74 & 70.02 \\
\hline 821 & 320 & 5.70 & 65 & & 352 & 1.86 & 72.20 \\
\hline $9_{1}$ & 376 & 76.01 & 76.43 & $9_{44}$ & 352 & 71.89 & 72.23 \\
\hline $9_{2}$ & 392 & 79.57 & 79.92 & $9_{45}$ & 368 & 75.16 & 75.51 \\
\hline$\overline{9_{3}}$ & 392 & 78.71 & 79.05 & $9_{46}$ & 344 & 69.09 & 69.35 \\
\hline 9 & 384 & 78.54 & 78.84 & $9_{47}$ & 368 & 75.29 & 75.61 \\
\hline 95 & 392 & 79.95 & 80.32 & 94 & 368 & 74.58 & 74.92 \\
\hline 96 & 400 & 80.30 & 80.65 & $9_{49}$ & 368 & 74.14 & 74.50 \\
\hline S & 400 & 82.10 & 82.65 & & & & \\
\hline
\end{tabular}

TABLE 1. Knot type, number of edges used, polygonal ropelength, and computed upper bound for the smooth ropelength.

tex in the positive and negative $x, y$, and $z$ directions. The minimizing algorithm perturbs the vertices in each of these directions and accepts knots with decreasing ropelength. The size of the perturbation is slowly brought to zero. Failure to improve the ropelength under any of these perturbations insures that the knot is vertex- critical up to the maximum reliability of the computations. Thus, the final conformations provide reasonable approximations to ropelength minima.

Table 1 contains the knot type, number of edges used, polygonal ropelength of the conformation after the minimizing algorithm was run, and the computed upper bound for the ropelength of the inscribed smooth knot (which is an upper bound for the minimum ropelength of the knot type).

Notice that the difference between the polygonal ropelength and the upper bound is at most $\approx 1 / 2 \%$. The trefoil is included twice: with 160 edges and with 654 edges. The additional edges help the trefoil achieve both a lower polygonal ropelength and a smaller error $(\approx 0.003 \%)$.

\section{LIMITING BEHAVIOR}

One goal of this work is to justify the computer simulations with polygonal energies. One would like to minimize the energy of polygonal knots with increasing numbers of edges and say that the resulting energy values of the polygons converge to the minimal energy of the smooth knot type. In this section, we show that if one can find a sequence of ropelength minimizing polygons with increasing number of edges, then the limit of the ropelengths is the minimum smooth ropelength. Furthermore, a subsequence of the polygons converges to a smooth ropelength minimizing conformation. We also prove these results for a general smooth and polygonal energy pair which satisfies more stringent properties.

Finding an actual energy minimizing polygon is problematic. The finite precision of computers limits the accuracy of the computed energy values and the possible vertex positions. Furthermore, one cannot check whether a knot is actually in a global energy minimizing conformation.

To complicate matters, Calvo [Calvo 98] has shown that in the space of $n$-edge polygons, a knot type may have more than one path component. For example, in the space of six-edge polygonal knots, there are two components of right-handed trefoils. However, when more than one component of a knot type has been detected, the components have been related by dihedral actions on the numbering of the vertices, which does not result in knots with different energies. It has not been determined whether there exist distinct components within the same knot type that are not related by dihedral actions. Since the energy minimizations are done within one component, the computations may not discover the true minimal value in such a case. We believe that this 


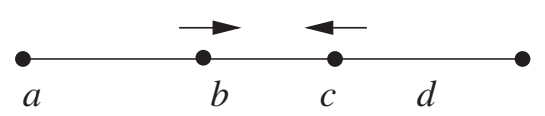

becomes

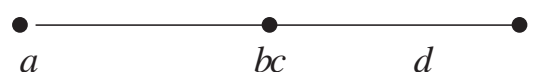

FIGURE 4. A portion of the knot collapses without affecting $\operatorname{Rad}(b)$ or $\operatorname{Rad}(c)$.

behavior is likely only an issue when the number of edges is near the minimal edge number or much of the knotting is contained in a portion of the knot with few edges, which is not the case here.

Thus, in practice, one can only guarantee that the computation has yielded a vertex-critical knot, up to the maximum reliability of the computer, that appears to be near a global minimum. Our polygons simply serve as comparison curves that let us estimate the minimum ropelength of smooth curves. Still, the results of different studies (using different minimization algorithms and different starting configurations) [Katritch et al. 96, Katritch et al. 97, Stasiak et al. 98, Millett and Rawdon 03] are fairly consistent and we assume our polygons are near optima.

Ropelength minimized polygons with many edges yield smooth knots with the smallest error in Theorem 3.2. Asymptotically, we show the error tends to zero in Theorem 8.3.

The space of polygonal knots explored in computations is $G e o(n)$, an open subspace of $\mathbb{R}^{3 n}$ consisting of all $n$-edge nonsingular polygonal knots whose first vertex is the origin. We cannot guarantee that a ropelength minimizing conformation exists in each component of $G e o(n)$. Consider a sequence of polygons with four collinear points converging to a polygon with three collinear points, the middle of which is a double point (see Figure 4). Since the turning angles at $b$ and $c$ are $0, \operatorname{Rad}(b)=\operatorname{Rad}(c)=\infty$ throughout the deformation. The conformation with the double point is not in $\operatorname{Geo}(n)$ and is a point of discontinuity of polygonal ropelength (in thinking of $\rho$ as a function on $\mathbb{R}^{3 n}$ ), but could still be the limit of decreasing ropelength polygons. Polygonal ropelength could be refined to incorporate such conformations, but it would make polygonal ropelength discontinuous. This behavior has not been observed in any of our calculations.

Even if we could show that each knot type and each number of edges yields a polygonal ropelength minimiz- ing conformation, this does not insure that the polygons converge to a smooth curve. Consider a sequence of minimizing conformations. It is possible that one or more pairs of adjacent edges could remain long (relative to the rest of the edges) throughout the sequence. If the angle between these edges is bounded away from zero, this would keep $\left(\sec \left(\frac{\theta_{\max }}{2}\right)-1\right)$, and thus, the error of Theorem 3.2 from approaching zero. We need that $\theta_{\max }$ tends to zero in the sequence. By bounding the ratio of the longest edge length to the shortest edge length, these issues disappear.

Definition 8.1. For a given polygonal knot $P$, let MaxEdge and MinEdge be the maximum and minimum edge lengths of $P$. Let $\mathrm{Geo}_{d}(n), d \geq 1$, be the subspace of Geo(n) consisting of polygons with $\frac{\text { MaxEdge }}{\text { MinEdge }} \leq d$.

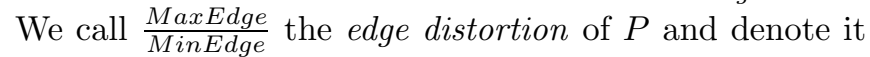
EdgeDistor $(P)$. For polygons $P$ and $Q$ with vertices $p_{i}$ and $q_{i}$ and turning angles $\theta_{i}$ and $\varphi_{i}$, respectively, define the norm on $\operatorname{Geo}(n)$ by

$$
\|P-Q\|=\max _{i}\left\|p_{i}-q_{i}\right\|+\max _{i}\left|\theta_{i}-\varphi_{i}\right| .
$$

Let $G_{e o}^{1}(n)$ be the subspace of $G_{d} o_{d}(n)$ consisting of polygons of total length 1 .

Note that $G_{e o}(n)$ consists of equilateral polygonal knots and $\operatorname{Geo}_{\infty}(n)=G e o(n)$.

Theorem 8.2. For any $1 \leq d<\infty$ and fixed $n$, each path component of $\mathrm{Geo}_{d}(n)$ contains a polygonal ropelength minimizing conformation.

Proof: Let $1 \leq d<\infty, n \in \mathbb{Z}^{+}$, and a path component $\mathcal{K} \in \mathrm{Geo}_{d}(n)$ be given. Since ropelength is scaleinvariant, we can restrict our study to $\operatorname{Geo}_{d}^{1}(n)$. The closure of $G e o_{d}^{1}(n)$ is compact in $\mathbb{R}^{3 n}$. Polygonal ropelength is continuous in $\operatorname{Geo}(n)$ with the norm described above [Rawdon 00], and thus, also in $G_{e o}^{1}(n)$. Hence, polygonal ropelength must attain a minimal value on the closure of $\mathrm{Geo}_{d}^{1}(n)$. We must insure that the minimum does not occur at a singular polygon.

Suppose $P$ is a singular polygonal knot lying in the closure of $\operatorname{Geo}_{d}^{1}(n)$. Since the edge distortion of $P$ is bounded by $d$, there are no consecutive double (or more) vertices. So the polygon $P$ must have two edges intersecting. If the two edges are consecutive, then any sequence of polygons converging to $P$ has MinRad $\rightarrow 0$. If the edges are nonconsecutive, then any sequence of polygons converging to $P$ has $d c s d \rightarrow 0$. In both cases, the rope- 
length becomes infinite and $P$ could not be a ropelength minimum.

In the space of $C^{1,1}$ knots, [Cantarella et al. 02, Gonzalez et al. 02, Gonzalez and de la Llave 03] show that ropelength minima exist for each knot type. For a given knot type, we have several knots of interest:

- A unit-length ropelength minimizing $C^{1,1}$ conformation $K^{\text {opt }}$ with arclength parameterization $f$ : $[0,1] \rightarrow \mathbb{R}^{3}$.

- The sequence of inscribed polygons $P_{n}^{\text {opt }}$ with vertices $\left\{f(0), f\left(\frac{1}{n}\right), \cdots, f\left(\frac{(n-1)}{n}\right)\right\}$.

- A sequence of polygonal ropelength minimizing conformations $P_{n}^{d}$, for any $1 \leq d<\infty$.

- The sequence of $C^{1,1}$ knots inscribed in $P_{n}^{d}$ via Proposition 3.1, which we call $K_{n}^{d}$.

We show that in the limit, the ropelength values of all of these knots are identical.

Theorem 8.3. For a given knot type and any $1<d<\infty$,

$$
\lim _{n \rightarrow \infty} \rho\left(P_{n}^{d}\right)=\lim _{n \rightarrow \infty} \rho\left(K_{n}^{d}\right)=\lim _{n \rightarrow \infty} \rho\left(P_{n}^{o p t}\right)=\rho\left(K^{o p t}\right) .
$$

Proof: Let $1<d<\infty$ and a knot type $\mathcal{K}$ be given. Define $K^{o p t}, P_{n}^{o p t}, P_{n}^{d}$, and $K_{n}^{d}$ as above. For this proof, we normalize all these conformations such that $\operatorname{Length}\left(K^{o p t}\right)=1$ and $\operatorname{Length}\left(P_{n}^{d}\right)=1$. Thus, for the families of inscribed knots $P_{n}^{\text {opt }}$ and $K_{n}^{d}$, we have Length $\left(P_{n}^{o p t}\right)<1$ and Length $\left(K_{n}^{d}\right)<1$.

The proof of the theorem is:

$$
\begin{aligned}
\lim _{n \rightarrow \infty} \rho\left(P_{n}^{d}\right) & \leq \lim _{n \rightarrow \infty} \rho\left(P_{n}^{o p t}\right) \\
& =\rho\left(K^{\text {opt }}\right) \\
& \leq \lim _{n \rightarrow \infty} \rho\left(K_{n}^{d}\right) \\
& =\lim _{n \rightarrow \infty} \rho\left(P_{n}^{d}\right) .
\end{aligned}
$$

We handle each of the inequalities and equalities separately, although there is some overlap between the arguments.

To prove $(8-1)$, note that for $n \geq \frac{\rho\left(K^{o p t}\right)}{\pi}, P_{n}^{o p t}$ has the same knot type as $K^{\text {opt }}$ by [Litherland et al. 99]. For $n$ sufficiently large, we show that the edge distortion of $P_{n}^{o p t}$ is at most $d$. The curvature of $K^{o p t}$ is bounded above by $\rho\left(K^{o p t}\right)$. If $n \geq \frac{\rho\left(K^{o p t}\right)}{\pi}$, then the arclength between any two vertices of $P_{n}^{o p t}$ (on $K^{o p t}$ ) is $\leq \pi R\left(K^{o p t}\right) \leq \pi M i n R a d\left(K^{o p t}\right)$. Thus, by Schur's Theorem, the chord distance between consecutive vertices of $P_{n}^{o p t}$ (i.e., the length of an edge of $P_{n}^{o p t}$ ) is bounded below by the distance between a pair of points of a circle of radius $R\left(K^{o p t}\right)$ with the same arclength. In other words,

$$
\operatorname{MinEdge}\left(P_{n}^{o p t}\right) \geq 2 R\left(K^{o p t}\right) \sin \frac{1}{2 n R\left(K^{o p t}\right)} .
$$

Since chord length exceeds arclength,

$$
\operatorname{Max} \operatorname{Edge}\left(P_{n}^{o p t}\right) \leq \frac{1}{n} .
$$

Thus,

$$
\text { EdgeDistor }\left(P_{n}^{o p t}\right) \leq \frac{1}{2 n R\left(K^{o p t}\right) \sin \frac{1}{2 n R\left(K^{o p t}\right)}}
$$

and as $n \rightarrow \infty$, EdgeDistor $\left(P_{n}^{o p t}\right) \rightarrow 1$. In particular, for large enough $n$, EdgeDistor $\left(P_{n}^{o p t}\right) \leq d$.

Thus, for sufficiently large $n, P_{n}^{\text {opt }}$ has the same knot type as $P_{n}^{d}$ in $\operatorname{Geo}^{d}(n)$ and since $P_{n}^{d}$ is a ropelength minimum from $G e o^{d}(n)$, we know that $\rho\left(P_{n}^{d}\right) \leq \rho\left(P_{n}^{o p t}\right)$. This concludes the proof of $(8-1)$.

The equality (8-2) is a result from [Rawdon 98, Rawdon 00].

For (8-3), we want to use Theorem 6.1 to say that $K_{n}^{d}$ has the same knot type as $P_{n}^{d}$ and, thus, we must have $\rho\left(K^{o p t}\right) \leq \rho\left(K_{n}^{d}\right)$. We must first show that $\theta_{\max }^{n}$ (i.e., $\theta_{\max }$ on $\left.P_{n}^{d}\right)$ is smaller than $2 \operatorname{arcsec}\left(\frac{3}{2}\right)$ for sufficiently large $n$. In the following claim, we prove a stronger result which we need for $(8-4)$.

Claim 1: $\theta_{\max }^{n} \rightarrow 0$ as $n \rightarrow \infty$

Proof of Claim 1: For the polygon $P_{n}^{d}$,

$$
R\left(P_{n}^{d}\right) \leq \operatorname{MinRad}\left(P_{n}^{d}\right) \leq \frac{\left|e_{i}\right|}{2 \tan \left(\frac{\theta_{\max }^{n}}{2}\right)},
$$

where $e_{i}$ is the shorter of the edges adjacent to the vertex realizing $\theta_{\max }^{n}$ on $P_{n}^{d}$. In particular,

$$
R\left(P_{n}^{d}\right) \tan \left(\frac{\theta_{\max }^{n}}{2}\right) \leq \frac{\operatorname{Max} E d g e\left(P_{n}^{d}\right)}{2}
$$

Now $(n-1) \operatorname{MinEdge}\left(P_{n}^{d}\right)+\operatorname{MaxEdge}\left(P_{n}^{d}\right) \leq$ $\operatorname{Length}\left(P_{n}^{d}\right)$. Since $\frac{\operatorname{MaxEdge}\left(P_{n}^{d}\right)}{\operatorname{MinEdge}\left(P_{n}^{d}\right)} \leq d$, we know that

$$
\operatorname{Max} \operatorname{Edge}\left(P_{n}^{d}\right) \leq \frac{d \operatorname{Length}\left(P_{n}^{d}\right)}{n+d-1} .
$$

We use this fact again later in the proof. 
For the sake of this claim, we know $\operatorname{Length}\left(P_{n}^{d}\right)=$ 1 for each $n$ and $d$ is a fixed finite number. Thus $\operatorname{MaxEdge}\left(P_{n}^{d}\right) \rightarrow 0$ as $n \rightarrow \infty$.

If we can bound $R\left(P_{n}^{d}\right)$ from below, then $\theta_{\max }^{n} \rightarrow 0$ by (8-5). For sufficiently large $n, R\left(P_{n}^{o p t}\right)$ is bounded below and $P_{n}^{o p t}$ is an element of $\operatorname{Geo}_{d}(n)$. Since $P_{n}^{o p t}$ is inscribed in $K^{\text {opt }}, \operatorname{Length}\left(P_{n}^{\text {opt }}\right)<\operatorname{Length}\left(K^{\text {opt }}\right)=1=$ $\operatorname{Length}\left(P_{n}^{d}\right)$. Since $\rho\left(P_{n}^{d}\right)$ is minimal, $R\left(P_{n}^{d}\right)>R\left(P_{n}^{\text {opt }}\right)$ and $R\left(P_{n}^{d}\right)$ is bounded below for sufficiently large $n$.

Thus, $\theta_{\max }^{n} \rightarrow 0$ as $n \rightarrow \infty$. This concludes the proof of Claim 1.

We proceed to $(8-4)$. We do this in two parts: First, we show Length $\left(K_{n}^{d}\right) / \operatorname{Length}\left(P_{n}^{d}\right) \rightarrow 1$ and then show $R\left(K_{n}^{d}\right) / R\left(P_{n}^{d}\right) \rightarrow 1$.

Claim 2: $\quad \frac{\text { Length }\left(K_{n}^{d}\right)}{\text { Length }\left(P_{n}^{d}\right)} \rightarrow 1$.

Proof of Claim 2: For a fixed $n$, the polygon $P_{n}^{d}$ and $K_{n}^{d}$ agree on some (possibly nonexistent) straight segments, but $K_{n}^{d}$ will be shorter near the vertices. At a vertex $v$, suppose $e$ is the shorter of the adjacent edges. Then the length of the arc of $K_{n}^{d}$ inscribed near $v$ is $(R(P)$ angle $(v))$. The length of $P_{n}^{d}$ in the same region is $\left(2 R(P) \tan \left(\frac{\text { angle }(v)}{2}\right)\right)$. The greatest difference between the length of $P_{n}^{d}$ and the length of $K_{n}^{d}$ occurs when the turning angle is largest. Thus,

$$
\begin{aligned}
\operatorname{Length}\left(K_{n}^{d}\right) \geq \operatorname{Length}\left(P_{n}^{d}\right)-n R\left(P_{n}^{d}\right) \\
\quad \times\left(2 \tan \left(\frac{\theta_{\max }^{n}}{2}\right)-\theta_{\max }^{n}\right) .
\end{aligned}
$$

When $x<1$, one can show that $2 \tan (x / 2)-x \leq \frac{x^{3}}{10}$. Thus,

$$
\operatorname{Length}\left(K_{n}^{d}\right) \geq \operatorname{Length}\left(P_{n}^{d}\right)-n R\left(P_{n}^{d}\right)\left(\frac{\left(\theta_{\max }^{n}\right)^{3}}{10}\right) .
$$

Assume that $n$ is sufficiently large so that $\theta_{\max }^{n}<1$. We want to bound $n R\left(P_{n}^{d}\right) \theta_{\text {max }}^{n}$.

We have

$$
\begin{array}{rlr}
n R\left(P_{n}^{d}\right) \theta_{\max }^{n} & \leq 2 n R\left(P_{n}^{d}\right) \tan \left(\frac{\theta_{\max }^{n}}{2}\right) \\
& \leq n \operatorname{Max} \operatorname{Edge}\left(P_{n}^{d}\right) \quad \text { by }(8-5) \\
& \leq \operatorname{Length}\left(P_{n}^{d}\right) \frac{n d}{d+n-1} \quad \text { by }(8-6) \\
& <d \quad \text { since Length }\left(P_{n}^{d}\right)=1 .
\end{array}
$$

Thus,

$$
\operatorname{Length}\left(K_{n}^{d}\right) \geq \operatorname{Length}\left(P_{n}^{d}\right)-\frac{d}{10}\left(\theta_{\max }^{n}\right)^{2} .
$$

We also know that $\operatorname{Length}\left(K_{n}^{d}\right) \leq \operatorname{Length}\left(P_{n}^{d}\right)$. Dividing by Length $\left(P_{n}^{d}\right)$ (which has been normalized to be 1), we get

$$
1-\frac{d\left(\theta_{\max }^{n}\right)^{2}}{10} \leq \frac{\operatorname{Length}\left(K_{n}^{d}\right)}{\operatorname{Length}\left(P_{n}^{d}\right)} \leq 1 .
$$

Thus, $\lim _{n \rightarrow \infty} \frac{\operatorname{Length}\left(K_{n}^{d}\right)}{\operatorname{Length}\left(P_{n}^{d}\right)}=1$. This concludes the proof of Claim 2.

Claim 3: $\quad \lim _{n \rightarrow \infty} \frac{R(K)}{R(P)}=1$.

Proof of Claim 3: By Theorem 3.2, $2-\sec \left(\frac{\theta_{\max }^{n}}{2}\right) \leq$ $\frac{R(K)}{R(P)} \leq 1$. Thus, $\lim _{n \rightarrow \infty} \frac{R(K)}{R(P)}=1$. This concludes the proof of Claim 3.

By Claims 2 and 3,

$$
\begin{aligned}
\lim _{n \rightarrow \infty} \rho\left(P_{n}^{d}\right) & =\lim _{n \rightarrow \infty} \frac{\operatorname{Length}\left(P_{n}^{d}\right)}{R\left(P_{n}^{d}\right)}=\lim _{n \rightarrow \infty} \frac{\operatorname{Length}\left(K_{n}^{d}\right)}{R\left(K_{n}^{d}\right)} \\
& =\lim _{n \rightarrow \infty} \rho\left(K_{n}^{d}\right)
\end{aligned}
$$

as desired.

The proof of Theorem 8.3 should generalize to other energy functions. We include a proof here for a class of energy functions. The ropelength energy does not satisfy hypothesis (2) below and hypothesis (3) of Theorem 8.4, which is why we did the analysis with $\mathrm{Geo}_{d}(n)$.

Let $\mathbb{K}$ be a space of knots. Following the definitions from [Diao et al. 98b], a function $E: \mathbb{K} \rightarrow \mathbb{R}$ is a charge energy function if it satisfies the following properties:

1. $E$ is continuous.

2. $E$ tends to infinity for any sequence of knots converging to a singular knot.

3. $E$ is invariant under scale changes and rigid motions of space.

Note that the Möbius energy does not satisfy the second condition of charge energy function because of its Möbius invariance. We can then state a generalization of Theorem 8.3.

Theorem 8.4. If $E$ is a charge energy function and

1. a smooth energy optimum, $K^{\text {opt }}$, exists for each knot type,

2. $E\left(P_{n}^{o p t}\right) \rightarrow E\left(K^{o p t}\right)$, where $P_{n}^{o p t}$ is the polygon whose vertices are equally distributed with respect to arclength on $K^{\text {opt }}$, 
3. within each path component of $G e o(n)$, a polygonal energy optimum, $P_{n}$, exists,

4. for sufficiently large $N$ and all $n \geq N$, there exists a smooth knot $K_{n}$ such that $E\left(K_{n}\right) \rightarrow E\left(P_{n}\right)$,

then

$\lim _{n \rightarrow \infty} E\left(P_{n}\right)=\lim _{n \rightarrow \infty} E\left(K_{n}\right)=\lim _{n \rightarrow \infty} E\left(P_{n}^{o p t}\right)=E\left(K^{o p t}\right)$.

Proof: We have $E\left(P_{n}\right) \leq E\left(P_{n}^{o p t}\right)$ and $E\left(K^{o p t}\right) \leq$ $E\left(K_{n}\right)$. Thus,

$$
\begin{aligned}
\lim _{n \rightarrow \infty} E\left(P_{n}\right) & \leq \lim _{n \rightarrow \infty} E\left(P_{n}^{o p t}\right)=E\left(K^{o p t}\right) \\
& \leq \lim _{n \rightarrow \infty} E\left(K_{n}\right)=\lim _{n \rightarrow \infty} E\left(P_{n}\right) .
\end{aligned}
$$

Finally, we show that a sequence of ropelength minimizing polygons has a subsequence that converges to a ropelength minimizing smooth knot. The following is a reworking of Dai and Diao's proof in [Dai and Diao 00]. They showed that under stronger conditions, a subsequence of the polygonal minimizing conformations of any polygonal energy must converge to a $C^{1}$ knot.

Theorem 8.5. Let $1<d<\infty$ and any knot type be given. If $\left\{P_{n}^{d}\right\}$ is a sequence of ropelength minimizing conformations in $\mathrm{Geo}_{d}^{1}(n)$ (for sufficiently large $n$ ), then there is a subsequence $\left\{P_{n_{k}}^{d}\right\}$ such that $P_{n_{k}}^{d} \rightarrow K^{\text {opt }}$ in the $C^{0}$ topology, where $K^{\text {opt }}$ is a ropelength minimizing $C^{1,1}$ conformation.

Proof: Let $\mathcal{C}^{1}$ consist of all $C^{1}$ arclength parameterizations of unit-length knots with $f(0)=(0,0,0)$ and

$B(m)=\left\{f: f \in \mathcal{C}^{1},\left\|\frac{f^{\prime}(t+h)-f^{\prime}(t)}{h}\right\| \leq m, \forall h \neq 0\right\}$.

Dai and Diao showed that $B(m)$ is a compact subset of $\mathcal{C}^{1}$. Let $K_{n}^{d}$ be the $C^{1,1}$ knot inscribed in $P_{n}^{d}$ via Proposition 3.1, normalized to length one. Let $f_{n}$ be an arclength parameterization of $K_{n}^{d}$ and $m=\sup \rho\left(P_{n}^{d}\right)$. Since $\rho\left(P_{n}^{d}\right)$ converges to $\rho\left(K^{o p t}\right)$, the constant $m$ is finite. Since $1 / R\left(P_{n}^{d}\right)=1 / \operatorname{MinRad}\left(K_{n}^{d}\right)=\operatorname{dil}\left(f^{\prime}\right) \leq \rho\left(K_{n}^{d}\right) \leq m$, each $f_{n} \in B(m)$. Thus, there exists a subsequence $\left\{f_{n_{k}}\right\}$ that converges to some $f_{0} \in \mathcal{C}^{1}$ under the $C^{1}$ norm. Let $K_{0}$ be the $C^{1}$ knot associated with $f_{0}$. It is shown in [Kusner and Sullivan 98, Cantarella et al. 02] that ropelength is lower semicontinuous with respect to the $C^{0}$ topology on the space of $C^{0,1}$ knots. Thus,

$$
\rho\left(K_{0}\right) \leq \lim _{n \rightarrow \infty} \rho\left(K_{n}^{d}\right)
$$

But Theorem 8.3 says that $\lim _{n \rightarrow \infty} \rho\left(K_{n}^{d}\right)=\rho\left(K^{o p t}\right)$. Thus, $\rho\left(K_{0}\right)=\rho\left(K^{o p t}\right)$ and $K_{0}$ must be a ropelength minimum. With respect to the $C^{0}$ topology, Proposition 3.1 tells us that $\left\|P_{n}^{d}-K_{n}^{d}\right\| \rightarrow 0$. Thus, $P_{n}^{d} \rightarrow K_{0}$ in the $C^{0}$ topology.

We can prove a similar result for a general energy function. We need a definition from [Dai and Diao 00].

Definition 8.6. An energy function $E$ defined on $C^{1,1}$ knots is tight if $E(K)>\frac{M}{\operatorname{MinRad}(K)}$ for some constant $M>0$.

Theorem 8.7. If in addition to the hypotheses of Theorem 8.4, $\left\|P_{n}-K_{n}\right\| \rightarrow 0$ in a $C^{0}$ sense and $E$ is a tight and charge energy, then there exists a subsequence $\left\{P_{n_{k}}\right\}$ such that $P_{n_{k}} \rightarrow K^{o p t}$ in a $C^{0}$ sense.

Proof: The requirement that $E$ is tight guarantees that the $K_{n}$ lie in $B(m)$ for some $m$. This rest of the argument is similar to the proof of Theorem 8.5.

\section{DISCUSSION}

To be a proper discretization of a smooth knot energy, the minimum energies of polygons should converge to the minimum energy of the smooth knot type. One would hope that the minimal energy polygonal conformations (or a subsequence) would converge to a smooth energy minimum as well. We have shown that polygonal ropelength adequately discretizes smooth ropelength. This behavior should not be unique to ropelength, although it will likely be more difficult to bound the energy of an inscribed smooth curve in terms of the energy of a polygon for other energies. This is an area where further research for other discrete energies would be valuable in order to put a solid foundation under all of the computer simulations we and others are doing. Other inscribing algorithms may be more fruitful in such analysis.

\section{ACKNOWLEDGMENTS}

The author thanks Robert Kusner, Kenneth Millett, and Jonathan Simon for helpful discussions and Piotr Pierański for providing SONO minimized knots and for many interesting discussions about polygonal ropelength functions. The author also thanks Michael Piatek for his work on the computations. This material is based upon work supported by the National Science Foundation under Grant No. 0074315 and 0296098. 


\section{REFERENCES}

[Buck and Orloff 95] Gregory Buck and Jeremey Orloff. "A Simple Energy Function for Knots." Topology Appl. 61:3 (1995), 205-214.

[Calvo 98] J. A. Calvo. Geometric Knot Theory: The Classification of Spatial Polygons with a Small Number of Edges. PhD thesis, University of California, Santa Barbara, 1998.

[Chern 67] S. S. Chern. "Curves and Surfaces in Euclidean Space." In Studies in Global Geometry and Analysis, pp. 16-56. Englewood Cliifs, NJ: Prentice-Hall, 1967.

[Cantarella et al. 02] Jason Cantarella, Robert B. Kusner, and John M. Sullivan. "On the Minimum Ropelength of Knots and Links." Invent. Math. 150:2 (2002), 257-286.

[Cerf and Stasiak 00] Corinne Cerf and Andrzej Stasiak. "A Topological Invariant to Predict the Three-Dimensional Writhe of Ideal Configurations of Knots and Links." Proc. Natl. Acad. Sci. USA 97:8 (2000), 3795-3798 (electronic).

[Dai and Diao 00] Xingde Dai and Yuanan Diao. "The Minimum of Knot Energy Functions." J. Knot Theory Ramifications 9:6 (2000), 713-724.

[Diao et al. 97] Y. Diao, C. Ernst, and E. J. Janse van Rensburg. "Knot Energies by Ropes." J. Knot Theory Ramifications 6:6 (1997), 799-807.

[Diao et al. 98a] Y. Diao, C. Ernst, and E. J. Janse van Rensburg. "Knots with Minimal Energies." In Ideal Knots, pp. 52-69. River Edge, NJ: World Sci. Publishing, 1998.

[Diao et al. 98b] Y. Diao, C. Ernst, and E. J. Janse van Rensburg. "Properties of Knot Energies." In Topology and Geometry in Polymer Science, pp. 37-47. New York: Springer Verlag, 1998.

[Diao et al. 99] Y. Diao, C. Ernst, and E. J. Janse van Rensburg. "Thicknesses of Knots." Math. Proc. Cambridge Philos. Soc. 126:2 (1999), 293-310.

[Diao 03] Yuanan Diao. "The Lower Bounds of the Lengths of Thick Knots." J. Knot Theory Ramifications 12:1 (2003), $1-16$.

[Durumeric et al. 97] O. Durumeric, R. A. Litherland, E. Rawdon, and J. Simon. Thickness of Knots 2. Preprint, 1997.

[Gonzalez and de la Llave 03] O. Gonzalez and R. de la Llave. "Existence of Ideal Knots." J. Knot Theory Ramifications 12:1 (2003), 123-133.

[Gonzalez and Maddocks 99] Oscar Gonzalez and John H. Maddocks. "Global Curvature, Thickness, and the Ideal Shapes of Knots." Proc. Natl. Acad. Sci. USA 96:9 (1999), 4769-4773 (electronic).

[Gonzalez et al. 02] O. Gonzalez, J. H. Maddocks, F. Schuricht, and H. von der Mosel. "Global Curvature and Self-Contact of Nonlinearly Elastic Curves and Rods." Calc. Var. Partial Differential Equations 14:1 (2002), 29-68.
[Gromov 83] Mikhael Gromov. "Filling Riemannian Manifolds." J. Differential Geom. 18:1 (1983), 1-147.

[Hoidn et al. 02] Phoebe Hoidn, Robert B. Kusner, and Andrzej Stasiak. "Quantization of Energy and Writhe in Self-Repelling Knots." New J. Phys. 4:20 (2002), 1-11. (electronic).

[Katritch et al. 96] Vsevolod Katritch, Jan Bednar, Didier Michoud, Robert G. Scharein, Jacques Dubochet, and Andrzej Stasiak. "Geometry and Physics of Knots." $\mathrm{Na}$ ture 384:6605 (1996), 142-145.

[Kim and Kusner 93] Denise Kim and Rob Kusner. "Torus Knots Extremizing the Möbius Energy." Experiment. Math. 2:1 (1993), 1-9.

[Katritch et al. 97] V. Katritch, W. K. Olson, P. Pieranski, J. Dubochet, and A. Stasiak. "Properties of Ideal Composite Knots." Nature 388 (1997), 148-151.

[Kusner and Sullivan 98] Robert B. Kusner and John M. Sullivan. "On Distortion and Thickness of Knots." In Topology and Geometry in Polymer Science, pp. 67-78. New York: Springer Verlag, 1998

[Krötenheerdt and Veit 76] Otto Krötenheerdt and Sigrid Veit. "Zur Theorie massiver Knoten." Wiss. Beitr. Martin-Luther-Univ. Halle-Wittenberg Reihe M Math. 7 (1976), 61-74.

[Litherland et al. 99] R. A. Litherland, J. Simon, O. Durumeric, and E. Rawdon. "Thickness of Knots." Topology Appl. 91:3 (1999), 233-244.

[Milnor 50] J. W. Milnor. "On the Total Curvature of Knots." Ann. of Math. (2) 52 (1950), 248-257.

[Millett and Rawdon 03] K. C. Millett and Eric J. Rawdon. "Energy, Ropelength, and Other Physical Aspects of Equilateral Knots." J. Comput. Phys. 186:2 (2003), 426456.

[O'Hara 92a] Jun O'Hara. "Energy Functionals of Knots." In Topology Hawaii, pp. 201-214. River Edge, NJ: World Sci. Publishing, 1992.

[O'Hara 92b] Jun O'Hara. "Family of Energy Functionals of Knots." Topology Appl. 48:2 (1992), 147-161.

[O'Hara 98] J. O'Hara. "Energy of Knots." In Ideal Knots, pp. 288-314. River Edge, NJ: World Sci. Publishing, 1998.

[Pierański 97] Piotr Pierański. "Searching for Ideal Knots." Pro Dialog 5 (1997), 111-120, (in Polish).

[Pierański 98] Piotr Pierański. "In Search of Ideal Knots." In Ideal Knots, pp. 20-41. River Edge, NJ: World Sci. Publishing, 1998.

[Pierański and Przbyl 01] P. Pieranski and S. Przbyl. "QuasiQuantization of Writhe in Ideal Knots." Eur. Phys. J. E 6 (2001), 117-121.

[Rawdon 98] Eric J. Rawdon. "Approximating the Thickness of a Knot." In Ideal Knots, pp. 143-150. River Edge, NJ: World Sci. Publishing, 1998. 
[Rawdon 00] Eric J. Rawdon. "Approximating Smooth Thickness." J. Knot Theory Ramifications 9:1 (2000), 113-145.

[Stasiak et al. 98] Andrzej Stasiak, Jacques Dubochet, Vsevolod Katritch, and Piotr Pieranski. "Ideal Knots and their Relation to the Physics of Real Knots." In Ideal Knots, pp. 1-19. River Edge, NJ: World Sci. Publishing, 1998.
[Stasiak et al 96] A. Stasiak, V. Katritch, J. Bednar, D. Michoud, and J. Dubochet. "Electrophoretic Mobility of DNA Knots." Nature 384 (1996), 122.

[Smutny and Maddocks 03] Jana Smutny and John H. Maddocks. Approximation of Space Curves by Biarcs. Preprint, 2003.

Eric J. Rawdon, Duquesne University, Department of Mathematics and Computer Science, Pittsburgh, PA 15282 (rawdon@mathcs.duq.edu)

Received July 15, 2002; accepted in revised form September 23, 2003. 